\title{
ESTUDO DA FRIABILIDADE DA SOJA EM UNIDADE PILOTO DE TRANSPORTE PNEUMÁTICO
}

\author{
V. S. R. OLIVEIRA ${ }^{1}$, M. L. PEREIRA ${ }^{1}$, A. R. MALAFATTI ${ }^{1}$, B.V.D. PEDRO ${ }^{1}$, V. B. \\ PEREZ $^{1}$, V.S. ROSA ${ }^{2}$, M.S. MORAES ${ }^{2}$, A.R. SANTOS ${ }^{2}$, D. MORAES JÚNIOR ${ }^{2}$ \\ ${ }^{1}$ Alunos de graduação, Universidade Santa Cecilia, Faculdade de Engenharia Química \\ ${ }^{2}$ Doutores orientadores, Universidade Santa Cecília e Universidade de São Paulo, \\ Departamento de Engenharia Química \\ E-mail para contato: victorsakai@ hotmail.com
}

\begin{abstract}
RESUMO - O transporte pneumático é uma operação unitária utilizada no deslocamento de material particulado como pellets de polietileno e grãos como soja, milho e feijão. O processo consiste na movimentação dos sólidos no interior de um duto, por meio de ar ou gás inerte. A friabilidade dos sólidos neste transporte é função principalmente da velocidade do gás e vazão das partículas em contato com as singularidades e dutos. O presente trabalho teve como objetivo avaliar a friabilidade da soja em uma unidade piloto de transporte pneumático. $\mathrm{O}$ equipamento utilizado consistiu de $6,13 \mathrm{~m}$ de duto reto de acrílico extrudado com $117 \mathrm{~mm}$ de diâmetro interno, um ciclone, duas curvas de $90^{\circ}$ e um tê saída de lado com e sem prolongamento. Foram realizados 90 ensaios para as vazões de soja de 95,$1 ; 368,5$ e 564,4 kg/h. O gás usado foi o ar e sua velocidade foi mantida em 12 $\mathrm{m} / \mathrm{s}$, mínima necessária para que o material permanecesse em suspensão ao ser transportado. Os resultados indicaram a menor quebra da soja utilizando a singularidade tê saída de lado com prolongamento de $90 \mathrm{~mm}$ na base do equipamento (formação de um colchão amortecedor). A menor vazão de soja apresentou maior quebra, cerca de $0,2 \%$ da massa total.
\end{abstract}

\section{INTRODUÇÃO}

O transporte de sólidos particulados (nas formas de grãos, pó ou brita), tem influenciado a economia mundial em diversos setores industriais como, na indústria química, farmacêutica, alimentícia e metalúrgica, chegando a atingir mais de $80 \%$ do custo total do processo (MORAES et. al., 2017).

A característica do transporte pneumático é deslocar material pelo interior de uma tubulação. Esses sólidos são movimentados por ar ou outro gás, através de um soprador, ventilador ou compressor (GOMIDE, 1983).

Há dois sistemas em uso: direto, quando o sólido passa através do ventilador, e indireto (Figura 1) quando o sólido é alimentado a jusante do ventilador. A alimentação do sólido pode ser realizada por meio de válvula rotativa, alimentador helicoidal ou tanque de sopragem (GOMIDE, 1983). 
Figura 1 - Alimentação indireta no transporte pneumático.

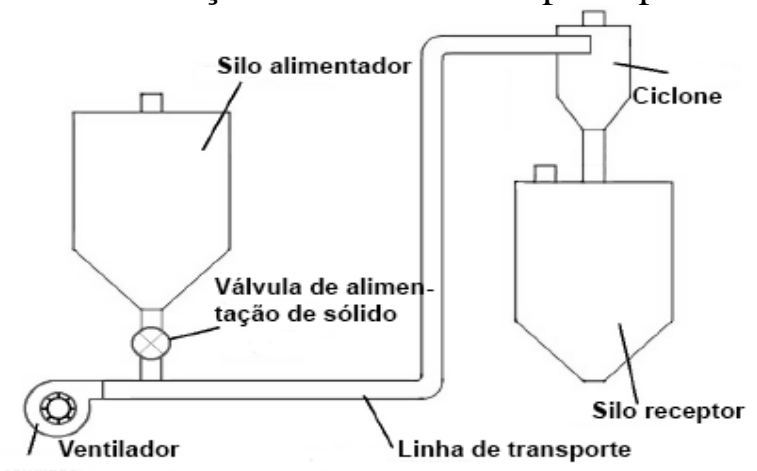

O transporte pneumático possui como vantagens: o baixo custo inicial, a facilidade de manutenção mecânica, a higiene e segurança para os trabalhadores, a mitigação da perda do material para o meio ambiente, o trajeto de transporte único ou ramificado, tornando-o por diversas vezes mais efetivo que outros transportadores (MORAES, 2012). Entretanto, o transportador pneumático pode requerer alta potência, transporte de produtos unidirecional, possibilidade de fragmentação significativa da partícula sólida, abrasão excessiva do duto, índice de ruído elevado em alta frequência e custo elevado para transportar partícula de grande diâmetro.

Pode-se classificar o transporte pneumático em fase densa e diluída. O transporte em fase diluída (Figura 2), mais empregado nas indústrias por não requerer mão de obra especializada para montagem e de menor custo inicial, caracteriza-se pelo transporte de baixa concentração de sólido em suspensão com o gás inerte, em velocidade acima de $10 \mathrm{~m} / \mathrm{s}$ e pressão menor que 15 psig.

Figura 2 - Tubulação de transporte pneumático em fase diluída.

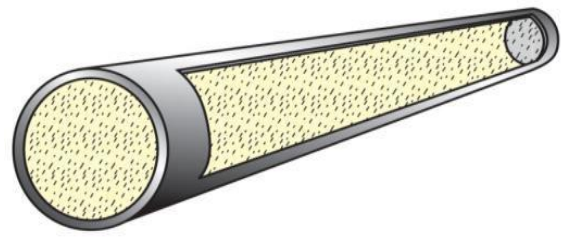

O transporte em fase densa (Figura 3) é caracterizado pela baixa quantidade de gás inerte para movimentar uma grande quantidade de sólidos particulados de forma pulsante, com concentração de partículas acima de $30 \%$ em volume, pressão acima de 15 psig e velocidade de gás em geral entre 1 e 5 m/s para não causar danos à tubulação ou as partículas (MORAES, 2012).

Figura 3 - Tubulação de transporte pneumático em fase densa.

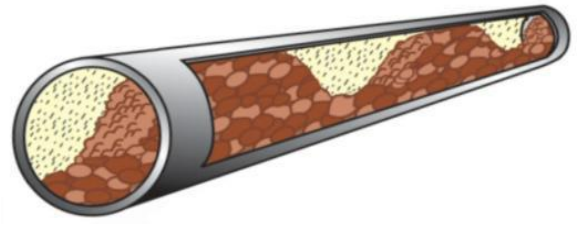

O estudo da friabilidade é de grande importância, pois o mesmo permite avaliar a resistência do material a ser transportado antes de inclúi-lo no processo industrial. 


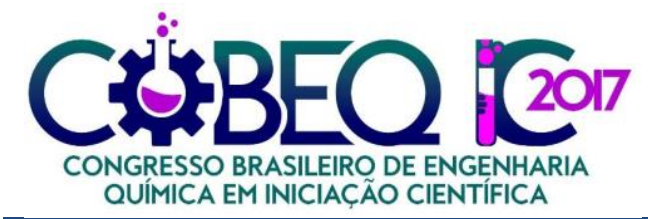

XII Congresso Brasileiro de Engenharia Química

em Iniciação Científica

UFSCar - São Carlos - SP

16 a 19 de Julho de 2017

\section{OBJETIVO}

O presente trabalho teve como objetivo avaliar a friabilidade da soja em uma unidade piloto de transporte pneumático em fase diluída, utilizando as singularidades "tê" saída lateral sem prolongamento, com prolongamento de $90 \mathrm{~mm}$, e curva com gomos para determinadas vazões de soja e com uma velocidade definida de ar.

\section{MATERIAL E MÉTODO}

Foi utilizada no presente estudo, uma unidade experimental de transporte pneumático (Figura 4) disponível no Laboratório de Operações Unitárias da Universidade Santa Cecília. O mesmo possuía um comprimento de duto reto de $6,13 \mathrm{~m}$, um ciclone de $0,32 \mathrm{~m}$ de diâmetro interno, uma curva de $90^{\circ}$ de quatro gomos fixa na parte superior do equipamento e uma intercalação das singularidades "tê" saída de lado e curva de três gomos (Figura 4, ponto 10). A soja empregada para o desenvolvimento do estudo foi da marca CAMIL.

Figura 4 - Unidade piloto de transporte pneumático e suas singularidades.

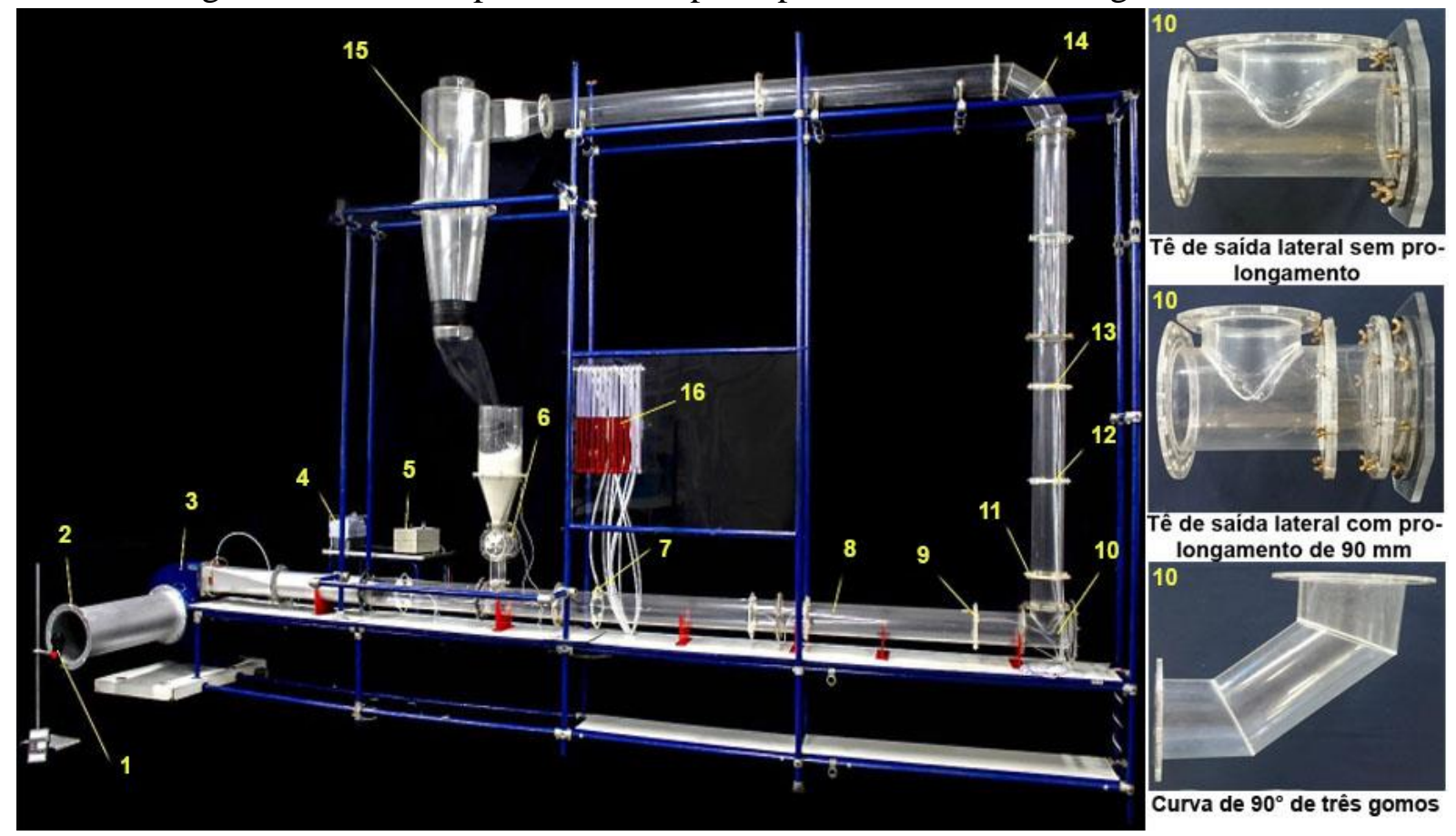

Os componentes da unidade piloto da Figura 4 são: 1- anemômetro (medidor de velocidade); 2- tubo de diâmetro interno de $217 \mathrm{~mm}$; 3- ventilador centrífugo; 4- inversor de frequência; 5- potenciômetro; 6- alimentação de sólido pelo silo; 7-tomadas piezométricas após a alimentação de sólido; 8- tubulação de acrílico de $117 \mathrm{~mm}$ de diâmetro interno; 9- tomadas piezométricas a montante da singularidade; 10 - singularidades removíveis; 11, 12 e 13- tomadas piezométricas a jusante da singularidade; 14 - curva de $90^{\circ}$ de quatro gomos; 15 - ciclone; 16 banco de manômetros de tubo em "U". A unidade não era automatizada, exigiu vários operadores para: a) controle da rotação do ventilador; b) controle da vazão de sólido; c) medida da velocidade do ar; d) acompanhamento das pressões; e) verificação da estanqueidade e f) 
troca das singularidades. O estudo exigiu a contagem manual dos grãos danificados em cada volta da unidade.

\subsection{Condições Operacionais}

A calibração da válvula rotativa para a determinação das vazões de soja, foi feita pelo método da massa por unidade de tempo. As vazões empregadas para o transporte da soja foram 95,092; 368,470 e 564,383 kg/h, com uma velocidade fixa de $12 \mathrm{~m} / \mathrm{s}$ na descarga do soprador, mínima necessária para que o material transportado permanecesse em suspensão.

Fixou-se as vazões mássicas de soja e realizou-se testes experimentais utilizando um anemômetro (Figura 4, item 1), para a medida da velocidade de sucção de ar. Após a determinação da área da sucção $\left(0,0370 \mathrm{~m}^{2}\right)$, recalque $\left(0,0104 \mathrm{~m}^{2}\right)$ e a velocidade de sucção $(3,5 \mathrm{~m} / \mathrm{s})$, foi obtida a velocidade de descarga pela Equação da Continuidade:

$$
\begin{aligned}
& v_{\text {suc }} \times A_{\text {suc }}=v_{\text {des }} \times A_{\text {des }} \\
& 3,5 \frac{\mathrm{m}}{\mathrm{s}} \times 0,0370 \mathrm{~m}^{2}=v_{\text {des }} \times 0,0108 \mathrm{~m}^{2} \\
& v_{\text {des }}=12,04 \frac{\mathrm{m}}{\mathrm{s}}
\end{aligned}
$$

\subsection{Ensaios}

Com uma massa inicial de soja seca de 7781,2 g, foram realizados 90 ensaios conforme as Tabelas 1, 2 e 3. Ao término de cada ensaio, coletou-se num recipiente a massa de soja que foi transportada, verificando-a numa balança com fundo de escala de $8 \mathrm{~kg}$ para determinar a quantidade de acúmulo que a singularidade "tê" saída de lado forneceu. Esse acúmulo foi o grande responsável pela redução da quebra do material ao deslocar por todo equipamento (efeito colchão amortecedor). O acúmulo foi quantificado por meio da diferença entre a massa inicial depositada no silo e a massa do recipiente que continha soja ao término de cada ensaio.

Após a coleta de massa e identificada a quantidade de acúmulo que os "tês" forneceram, foi distribuído todo o material do recipiente numa tela com suporte para fotografia, a fim de separar os grãos fragmentadas dos resistentes.

\section{RESULTADOS}

A partir das Tabelas 1,2 e 3, nota-se que a quebra da soja não agiu de uma maneira padronizada a cada ensaio. Houve uma oscilação de quebra, independente da singularidade trocada. Percebeu-se também que, com o aumento do prolongamento do tê saída de lado, houve um aumento de seu acúmulo em gramas (o efeito colchão amortecedor é maior) justificando sua eficiência. 
Tabela 1 - Friabilidade da soja nas singularidades e vazão de 95,092 kg/h.

\begin{tabular}{|c|c|c|c|c|c|c|c|c|c|c|c|}
\hline \multicolumn{12}{|c|}{ Teste de friabilidade com soja - Vazão de $95,092 \mathrm{~kg} / \mathrm{h}$} \\
\hline \multicolumn{12}{|c|}{ Curva $\left(T=24,5^{\circ} \mathrm{C}\right)$} \\
\hline Volta & $1^{\mathrm{a}}$ & $2^{\mathrm{a}}$ & $3^{\mathrm{a}}$ & $4^{\mathrm{a}}$ & $5^{\mathrm{a}}$ & $6^{\mathrm{a}}$ & $7^{\mathrm{a}}$ & $8^{a}$ & $9^{a}$ & $10^{\mathrm{a}}$ & Total \\
\hline m acún & - & - & - & - & - & - & - & - & - & - & - \\
\hline $\mathrm{m} \mathrm{q}$ & 2,60 & 2,30 & 0,90 & 0,80 & 1,50 & 1,00 & 1,90 &, 10 & 1,6 & 1,90 & 16,60 \\
\hline \multicolumn{12}{|c|}{ Sem prolongamento $\left(T=25^{\circ} \mathrm{C}\right)$} \\
\hline $\mathrm{V}$ & $1^{\mathrm{a}}$ & $2^{\mathrm{a}}$ & $3^{a}$ & $4^{a}$ & $5^{\mathrm{a}}$ & $6^{a}$ & $7^{a}$ & $8^{\mathrm{a}}$ & $9^{a}$ & $10^{\mathrm{a}}$ & Total \\
\hline $\mathrm{m}$ acúmulo $(\mathrm{g})$ & 544,8 & 545,5 & 570,3 & 559, & 554,4 & 588 & 544,1 & 555,0 & 569,9 & 553,9 & - \\
\hline m quebrado $(\mathrm{g})$ & 1,00 & 1,40 & 2,20 & 1,00 & 1,40 & 1,10 & 0,90 & 2,00 & 1,7 & 1,50 & 14,20 \\
\hline \multicolumn{12}{|c|}{ Prolongamento de $90 \mathrm{~mm}\left(\mathrm{~T}=25^{\circ} \mathrm{C}\right)$} \\
\hline Volta & $1^{a}$ & $2^{a}$ & $3^{a}$ & $4^{a}$ & $5^{a}$ & $6^{a}$ & $7^{a}$ & $8^{a}$ & $9^{a}$ & $10^{\mathrm{a}}$ & Total \\
\hline m acúmulo & 1088,6 & 1093,4 & 111,8 & 1072 & 1071,4 & 1058,1 & 1044,3 & 1020,8 & 1055,5 & 1052,8 & - \\
\hline $\mathrm{m}$ quebrado $(\mathrm{g})$ & 0,50 & 0,50 & 0,70 & 1,20 & 1,20 & 1,40 & 1,30 & 2,00 & 1,80 & 1,80 & 12,40 \\
\hline
\end{tabular}

Tabela 2 - Friabilidade da soja nas singularidades e vazão de 368,47 kg/h.

\begin{tabular}{|c|c|c|c|c|c|c|c|c|c|c|c|}
\hline \multicolumn{12}{|c|}{ Teste de friabilidade com soja - Vazão de $368,47 \mathrm{~kg} / \mathrm{h}$} \\
\hline \multicolumn{12}{|c|}{ Curva $\left(T=24,5^{\circ} \mathrm{C}\right)$} \\
\hline Volta & $1^{a}$ & $2^{a}$ & $3^{a}$ & $4^{\mathrm{a}}$ & $5^{\mathrm{a}}$ & $6^{a}$ & $7^{a}$ & $8^{\mathrm{a}}$ & $9^{a}$ & $10^{\mathrm{a}}$ & Total \\
\hline m acúmulo & - & 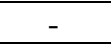 & - & - & 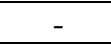 & - & - & - & - & 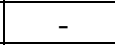 & 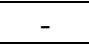 \\
\hline $\mathrm{m}$ queb & 1,00 & 0,90 & 1,10 & 1,40 & 2,10 & 0,90 & 1,60 & 0,90 & 2,00 & 1,90 & 13,80 \\
\hline \multicolumn{12}{|c|}{ Sem prolongamento $\left(\mathrm{T}=23^{\circ} \mathrm{C}\right.$} \\
\hline Volta & $1^{\mathrm{a}}$ & $2^{\mathrm{a}}$ & $3^{\mathrm{a}}$ & $4^{a}$ & $5^{a}$ & $6^{\mathrm{a}}$ & $7^{\mathrm{a}}$ & $8^{a}$ & $9^{a}$ & $10^{\mathrm{a}}$ & Total \\
\hline m acúmı & 670,0 & 655,4 & 654,8 & 642,7 & 653 & 648,1 & 639,4 & 650,3 & 650,3 & 647,9 & \\
\hline $\mathrm{m}$ quebrado $(\mathrm{g})$ & 0,80 & 1,00 & 1,40 & 1,30 & 1,00 & 0,90 & 1,50 & 2,00 & 2,10 & 1,10 & 13,10 \\
\hline \multicolumn{12}{|c|}{ Prolongamento de $90 \mathrm{~mm}\left(\mathrm{~T}=23^{\circ} \mathrm{C}\right)$} \\
\hline Volta & $1^{\mathrm{a}}$ & $2^{\mathrm{a}}$ & $3^{\mathrm{a}}$ & $4^{\mathrm{a}}$ & $5^{a}$ & $6^{a}$ & $7^{\mathrm{a}}$ & $8^{a}$ & $9^{a}$ & $10^{\mathrm{a}}$ & Total \\
\hline m acúmulo & 1296,4 & 1265,6 & 1244,3 & 297,5 & 279,0 & 1255,1 & 1245,5 & 1234,3 & 1239,5 & 1228,0 & - \\
\hline m quebrado $(\mathrm{g})$ & 1,00 & 0,50 & 0,30 & 0,40 & 0,10 & 0,10 & 0,80 & 0,40 & 0,50 & 0,30 & 4,40 \\
\hline
\end{tabular}

Tabela 3 - Friabilidade da soja nas singularidades e vazão de 564,383 kg/h.

\begin{tabular}{|c|c|c|c|c|c|c|c|c|c|c|c|}
\hline \multicolumn{12}{|c|}{ Teste de friabilidade com soja - Vazão de $564,383 \mathrm{~kg} / \mathrm{h}$} \\
\hline \multicolumn{12}{|c|}{ Curva $\left(T=24,5^{\circ} \mathrm{C}\right)$} \\
\hline Volta & $1^{\mathrm{a}}$ & $2^{\mathrm{a}}$ & $3^{\mathrm{a}}$ & $4^{\mathrm{a}}$ & $5^{\mathrm{a}}$ & $6^{\mathrm{a}}$ & $7^{\mathrm{a}}$ & $8^{\mathrm{a}}$ & $9^{a}$ & $10^{\mathrm{a}}$ & Total \\
\hline m acúmulo $(\mathrm{g})$ & 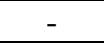 & - & - & - & - & - & - & - & - & 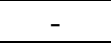 & - \\
\hline m quebrado $(\mathrm{g})$ & 1,80 & 1,00 & 0,60 & 0,60 & 1,00 & 0,60 & 1,50 & 1,60 & 1,30 & 1,30 & 11,30 \\
\hline \multicolumn{12}{|c|}{ Sem prolongamento $\left(\mathrm{T}=23^{\circ} \mathrm{C}\right)$} \\
\hline $\mathrm{Vc}$ & $1^{\mathrm{a}}$ & $2^{\mathrm{a}}$ & $3^{\mathrm{a}}$ & $4^{\mathrm{a}}$ & $5^{\mathrm{a}}$ & $6^{\mathrm{a}}$ & $7^{\mathrm{a}}$ & $8^{\mathrm{a}}$ & $9^{a}$ & $10^{\mathrm{a}}$ & Total \\
\hline m acún & 660,5 & 81,4 & 712,8 & 693, & 77( & 755, & 727,5 & 759,1 & 761,0 & 1047,8 & 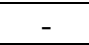 \\
\hline m quebrado $(\mathrm{g})$ & 3,20 & 0,80 & 0,40 & 0,70 & 0,60 & 0,40 & 0,90 & 1,00 & 0,90 & 0,8 & 9,70 \\
\hline \multicolumn{12}{|c|}{ Prolongamento de $90 \mathrm{~mm}\left(\mathrm{~T}=23^{\circ} \mathrm{C}\right)$} \\
\hline Volta & $1^{\mathrm{a}}$ & $2^{\mathrm{a}}$ & $3^{\mathrm{a}}$ & $4^{\mathrm{a}}$ & $5^{\mathrm{a}}$ & $6^{\mathrm{a}}$ & $7^{\mathrm{a}}$ & $8^{\mathrm{a}}$ & $9^{a}$ & $10^{\mathrm{a}}$ & Total \\
\hline $\mathrm{m}$ acúmulo $(\mathrm{g})$ & 1284,7 & 1298,2 & 1304,2 & 1303,7 & 1313,8 & 1315,1 & 1336,0 & 1341,1 & 1333,1 & 1329,2 & - \\
\hline m quebrado (g) & 0,50 & 0,80 & 0,50 & 0,40 & 0,60 & 0,20 & 0,40 & 0,40 & 0,30 & 0,50 & 4,60 \\
\hline
\end{tabular}

Conforme indicado na Figura 5, o "tê" saída lateral de prolongamento de $90 \mathrm{~mm}$ mostrouse mais efetivo na redução da quebra do material nas vazões de 95,092; 368,470 e 564,383 kg/h. A curva de $90^{\circ}$ de 2 gomos não possui acúmulo, e por isso houve um contato maior entre o sólido e a tubulação interna, gerando uma quantidade maior de quebra. O "tê" saída lateral sem prolongamento demonstrou valores intermediários. 
Figura 5 - Total de grãos de soja quebradas (ordenada) em relação as três vazões mássicas de soja (abcissas).

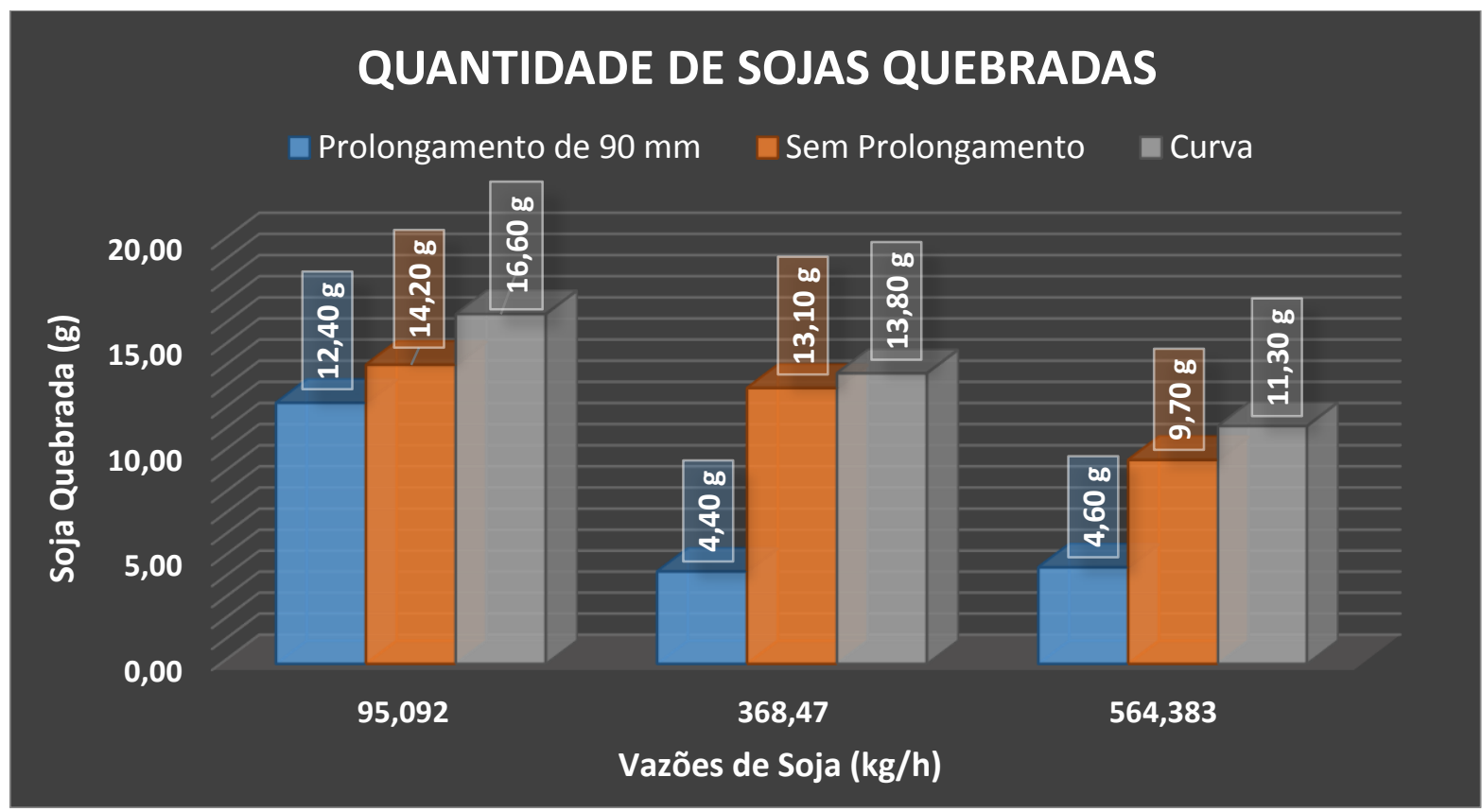

\section{CONCLUSÃO}

Concluiu-se que a friabilidade da soja, em relação à mudança de singularidade na parte inferior da unidade experimental (Figura 4, item 10), foi alterada. Conforme indicado na Figura 5, o "tê" saída lateral de prolongamento de $90 \mathrm{~mm}$ mostrou-se mais efetivo na redução da quebra do material nas vazões de 95,092; 368,470 e 564,383 kg/h, devido a uma maior quantidade de acúmulo que é formado em seu prolongamento, criando uma condição de colchão amortecedor. Com a maior vazão de grãos de soja $(564,383 \mathrm{~kg} / \mathrm{h})$ observou-se uma menor quebra em função do menor impacto com os dutos e singularidades.

\section{REFERÊNCIAS}

GOMIDE R.; Operações Unitárias. Transporte de sólidos, São Paulo, ed. Do autor. 1983, v. 1.

MORAES, M.S.; Tese de Doutorado: “Convecção forçada de partículas poliméricas em fase diluída: Curvas de pressão e distribuição de partículas”, UNICAMP, 2012.

MORAES, M.S., TORNEIROS, D.L.M., ROSA, V.S., HIGA, J.S., CASTRO, Y.R., SANTOS, A.R., COELHO, N.M.A., MORAES JUNIOR, D. Experimental quantification of the head loss coefficient $\mathrm{k}$ for fittings and semi-industrial pipe cross section solid concentration profile in pneumatic conveying of polypropylene pellets in dilute phase. Powder Technology $310 \mathrm{pg} 250$ 263, 2017. 